Mor et al., 2020- preprint version -www.biorxiv.org

\title{
Metabolic defects cause hyperactive mitochondria and Parkinson's disease-like traits
}

\author{
Danielle E. Mor ${ }^{1}$, Salman Sohrabi ${ }^{1}$, Rachel Kaletsky ${ }^{1}$, Will Keyes ${ }^{1}$, Vrinda Kalia ${ }^{2}$, Gary W. Miller ${ }^{2}$, Coleen T. \\ Murphy $^{1 *}$ \\ ${ }^{1}$ Department of Molecular Biology \& LSI Genomics, Princeton University, Princeton NJ 08544 \\ ${ }^{2}$ Department of Environmental Health Sciences, Mailman School of Public Health, Columbia University, New York \\ NY 10032 \\ *Corresponding Author: ctmurphy@princeton.edu
}

\begin{abstract}
$\underline{\text { Abstract }}$
Metabolic dysfunction is a facet of many age-related neurodegenerative diseases, yet its role in disease etiology remains poorly understood ${ }^{1}$. We recently discovered a potential causal link between the branchedchain amino acid transferase, $B C A T-1$, and the neurodegenerative movement disorder, Parkinson's disease $(\mathrm{PD})^{2}$. Knockdown of $\boldsymbol{C}$. elegans bcat-1 recapitulates PD-like features, including progressive motor deficits and neurodegeneration with age ${ }^{2}$. Using transcriptomic, metabolomic, and imaging approaches, we show that bcat-1 knockdown increases mitochondrial activity and induces oxidative damage in neurons through mTORindependent mechanisms. We recently developed a high-throughput screening platform to identify drugs that may be repurposed for $P D$, and found that metformin, the leading type 2 diabetes medication, significantly improves motor function in bcat-1(RNAi) worms ${ }^{3}$. Late-in-life metformin treatment restores normal mitochondrial activity levels and protects against bcat-1-associated neurodegeneration. Our results suggest that PD may originate as a metabolic disorder, and highlight metformin as a promising new drug candidate for PD treatment.
\end{abstract}

Parkinson's disease (PD) is a devastating neurodegenerative movement disorder, and its prevalence is predicted to significantly increase as the population ages, ${ }^{4,5}$ PD is characterized by the progressive loss of dopaminergic neurons in the substantia nigra, and the formation of pathological inclusions containing aggregated $\alpha$-synuclein protein ${ }^{6,7}$. The mechanisms that induce neurodegeneration in PD are still poorly understood, and greater than $90 \%$ of cases have no known cause ${ }^{8}$.

In an effort to identify novel PD genes, we recently developed diseaseQUEST, a method that combines human genome-wide association studies with tissue-specific functional genetic networks and high-throughput behavioral screening in $C$. elegans $^{2}$. Using this computational-experimental framework, we discovered a novel link between branched-chain amino acid transferase 1 (BCAT-1), which catalyzes the first step in branched-chain amino acid (BCAA) catabolism ${ }^{9}$, and PD. We found that $B C A T-1$ expression is normally high in PDsusceptible regions of the healthy human brain, and its expression is reduced in the substantia nigra of sporadic PD patients ${ }^{2}$. While these findings suggest a correlation between defective BCAA metabolism and PD, animal models are required to determine causality and underlying mechanisms.

The nematode C. elegans offers a unique system in which to study age-related neurological disease, due to its short lifespan and highly conserved nervous system signaling that gives rise to a set of complex behaviors. Moreover, the worm is highly amenable to high-throughput screening approaches, allowing rapid testing of potential disease treatment options ${ }^{10}$. Previously, we showed that RNAi-mediated reduction of neuronal bcat-1 in C. elegans causes progressive, agedependent motor dysfunction and accelerates dopamine neuron degeneration in worms expressing human $\alpha$ synuclein $^{2}$. Here, we use tissue-specific transcriptomics, highresolution metabolomics, and imaging in aged worms, revealing that bcat-1 knockdown - surprisingly - increases neuronal mitochondrial activity. The type 2 diabetes medication metformin restores normal mitochondrial activity levels, reduces neurodegeneration, and significantly improves motor function, even with late-in-life administration. Our work underscores the possibility that at least some forms of PD may primarily be a metabolic disorder, and offers metformin as a new potential treatment option for PD.

Neuronal transcriptional analysis reveals upregulation of bioenergetic pathways

To model the reduction of $B C A T-1$ that was observed in PD patient substantia nigra ${ }^{2}$, we performed adult-specific RNAimediated knockdown of bcat-1 in C. elegans to avoid embryonic/larval lethality ${ }^{11}$. Consistent with our previous findings ${ }^{2}$, bcat-1 knockdown exclusively in the nervous system (sid-1;Punc-119::sid-1) caused severe, spasm-like 'curling' motor dysfunction in aged (day 8 adult) animals (Fig. 1a). By this timepoint, dopamine neurons are almost entirely lost in neuronal RNAi-sensitive worms expressing dopaminergic-specific $\alpha$-synuclein (Extended Data Fig. 1a); however, examination at the earlier timepoint of day 6 
Mor et al., 2020 - preprint version -www.biorxiv.org

revealed that bcat-1 knockdown exacerbated the loss of cell bodies and the fragmentation and blebbing of neurites (Fig. 1b). Dopaminergic degeneration is associated with loss of $C$. elegans' basal slowing response, in which worms normally slow down in the presence of a bacterial food source ${ }^{12}$. Consistent with the observed structural damage (Fig. 1b), bcat-1 knockdown worsened basal slowing performance (Extended Data Fig. 1b).

To understand how bcat-1 reduction causes neurodegeneration and motor deficits, we first investigated the mTOR pathway, which was previously implicated in bcat-1 regulation of lifespan ${ }^{13}$. However, we found that this pathway does not interact with bcat-1 to regulate PD-related phenotypes, since

a

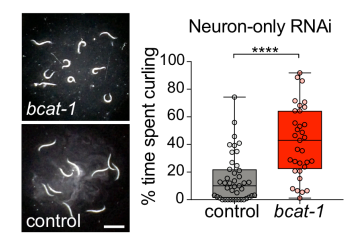

b

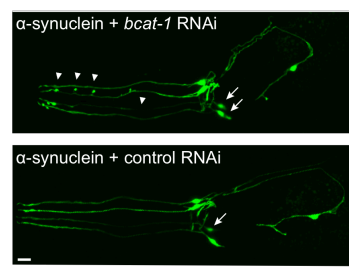

c

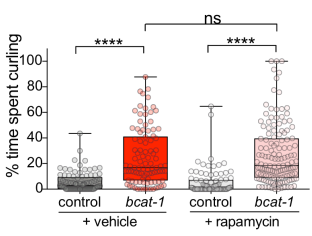

d
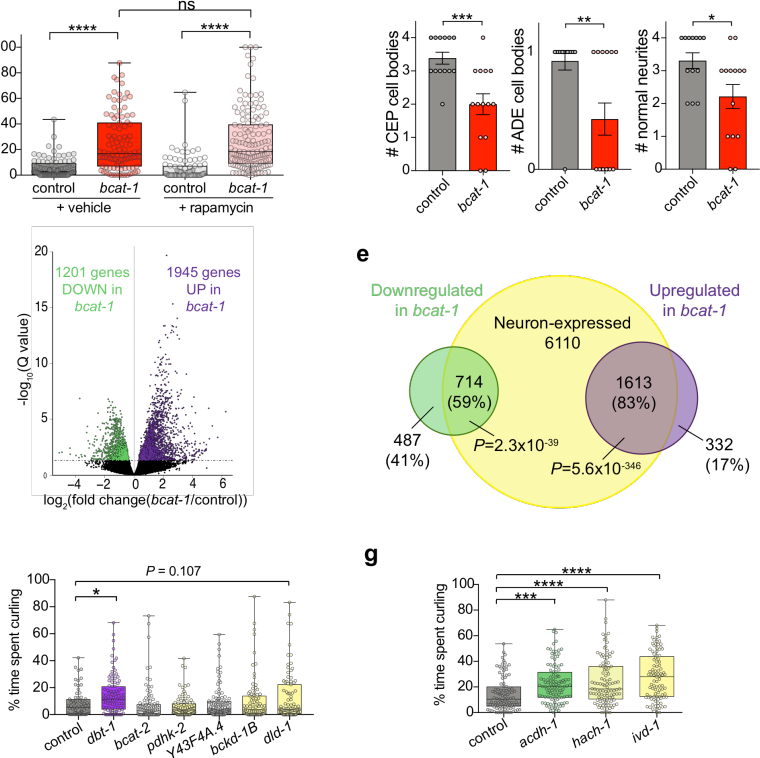

h
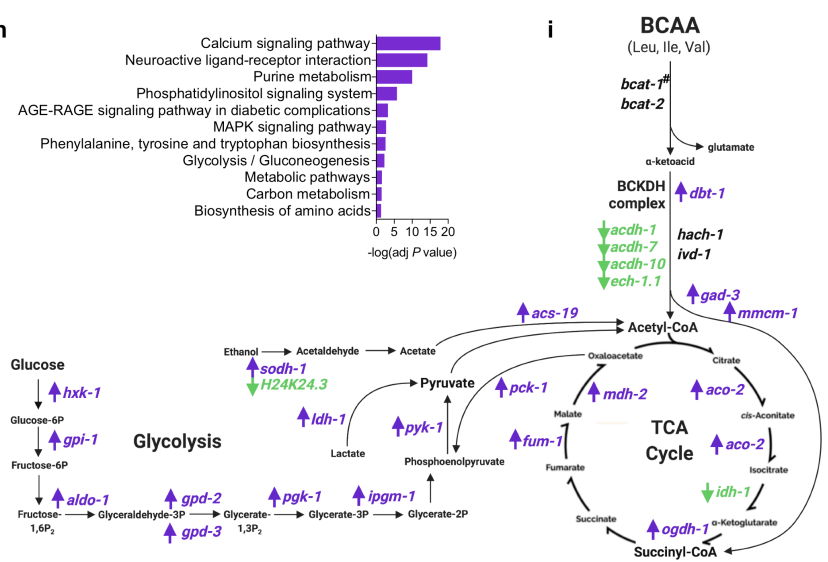

neither mTOR/let-363 RNAi nor rapamycin treatment had any effect on curling behavior (Fig. 1c, Extended Data Fig. 2).

Therefore, we decided to take an unbiased and tissue-specific approach to uncover the mechanisms of bcat-1 neurotoxicity, using our method for the isolation and RNA sequencing of adult $C$. elegans tissues ${ }^{14}$. Neuronal RNAi-sensitive $C$. elegans were treated with bcat-1 or control RNAi, and neurons were collected on day 5 of adulthood, a timepoint that precedes severe motor dysfunction (Fig. 1a), in order to shed light on mechanisms that drive disease rather than reporting transcriptional changes from dying neurons. bcat-1(RNAi) and control RNAi-treated samples were distinct (Extended Data Fig. 3a), and the majority of the differentially-expressed $($ FDR $<0.05)$ genes (Fig. 1d, Extended Data Table 1) were previously identified as neuronally-expressed in adult wildtype C. elegans $^{15}$ (Fig. 1e). Gene Ontology analysis also revealed that genes upregulated in bcat-1(RNAi) neurons were largely neuronal, including terms such as chemical synaptic transmission, synapse organization, and regulation of neurotransmitter levels (Extended Data Fig. 3b,c).

Figure 1: bcat-1 knockdown upregulates bioenergetic pathway genes in neurons. a, Adult- and neuron-specific RNAi-mediated bcat- 1 knockdown results in severe spasm-like 'curling' motor dysfunction in aged (day 8 adult) animals. Scale bar, $1 \mathrm{~mm} . \mathrm{n}=43$ for control, 33 for bcat-1(RNAi). Two-tailed $t$ test. b, In neuronal RNAi-sensitive worms expressing $\alpha$ synuclein in dopaminergic neurons, bcat-1 knockdown accelerates degeneration of CEP and ADE dopaminergic cell bodies (arrows) and neurites (arrowheads) on day 6. Scale bar, $10 \mu \mathrm{m} . \mathrm{n}=13$ for control, 14 for bcat-1(RNAi). Two-tailed $t$-test. Data are mean \pm s.e.m. c, Curling is unaffected by rapamycin treatment in neuronal RNAi-sensitive worms with bcat-1 knockdown on day 8. ns, not significant. $n=92$ for control vehicle, 97 for bcat- 1 vehicle, 87 for control rapamycin, 139 for bcat-1 rapamycin. Two-way ANOVA with Tukey's post-hoc. de, Neurons were isolated on day 5 from neuronal RNAi-sensitive worms with adult-specific bcat-1 knockdown and RNAsequenced. $\mathrm{n}=5$ independent collections/group. Volcano plot (d) showing upregulated (purple) and downregulated (green) genes in bcat-1 (RNAi) neurons (FDR $<0.05)$. Venn diagram (e) showing a majority of differentially-expressed genes are expressed in neurons. $P$ values: hypergeometric distributions. f-g, Adultspecific knockdown of BCAA pathway components in neuronal RNAi-sensitive worms showed disruption of $d b t-1$ (f), acdh-1, hach-1, or $i v d-1$ (g) causes curling on day $8 . \mathrm{n}=70$ and 99 for control in (f) and (g), respectively, 100 for $d b t-1,95$ for bcat-2, 91 for $p d h k-2,111$ for $Y 43 F 4 A .4,85$ for $b c k d-1 B, 90$ for $d l d-1$, 111 for $a c d h-1,107$ for hach-1, 96 for $i v d-1$. One-way ANOVA with Dunnett's post-hoc. $\mathbf{h}, \mathrm{KEGG}$ analysis of genes upregulated in bcat-1(RNAi) neurons. i, BCAA, glycolysis, and TCA cycle pathway schematic with genes upregulated (purple) and downregulated (green) in bcat-1(RNAi) neurons. " bcat-1 expression could not be measured due to RNA-sequencing of the bcat 1 RNAi. $* P<0.05, * * P<0.01, * * * P<0.001$, $* * * * P<0.0001$. Box-plots show minimum, 25th percentile, median, 75th percentile, maximum. 
Mor et al., 2020- preprint version -www.biorxiv.org

Several genes involved in BCAA metabolism were differentially expressed in response to bcat- 1 knockdown (Fig. 1i, Extended Data Table 1). To test if disrupting these BCAA pathway components produces motor dysfunction similar to bcat-1(RNAi), neuronal RNAi-sensitive worms were fed RNAi as adults and tested for curling on day 8. The acyl-CoA dehydrogenase $a c d h-1$ was the most downregulated neuronal gene $\left(\log _{2}\right.$ (fold-change $)=-2.44, \mathrm{p}$-adj $\left.=2.87 \times 10^{-5}\right)$, while $d b t-1$, which encodes the catalytic core of the branched chain $\alpha$ ketoacid dehydrogenase complex (BCKDHC), was increased in bcat-1(RNAi) neurons ( $\log _{2}$ (fold-change $)=0.55$, padj=0.04). Disruption of either $a c d h-1$ or $d b t-1$ was sufficient to induce curling (Fig. 1f,g). Since $a c d h-1$ functions in valine and isoleucine metabolism but not leucine ${ }^{16}$, we also tested hach-1, a hydrolase specific to valine, and ivd-1, a dehydrogenase specific to leucine, and in each case, knockdown was sufficient to produce curling (Fig. 1g), indicating that defects in the metabolism of any of the three BCAAs can produce motor dysfunction. Therefore, normal BCAA metabolism appears to be critical to neuronal maintenance with age.

KEGG functional analysis of genes upregulated in bcat1(RNAi) neurons suggested enrichment of several metabolic pathways, including glycolysis/gluconeogenesis, carbon metabolism, metabolic pathways, and purine metabolism (Fig. 1h, Extended Data Fig. 3d). We further examined carbon metabolism, in particular glycolysis and the TCA cycle, since BCAA metabolism is closely linked with these bioenergetic pathways $^{9}$ (Fig. 1i) and BCAT-1 localizes to mitochondria in C. elegans ${ }^{17}$. The majority $(19 / 25)$ of the differentially-

Figure 2: Depletion of TCA cycle metabolites coincides with increased mitochondrial activity in bcat-1(RNAi) worms. a-e, Neuronal RNAi-sensitive worms were collected for metabolomics on day 5 following adult-specific bcat-1 knockdown. Manhattan plot (a) and heat-map (b) show features increased (purple) and decreased (green) $(P<0.05)$ in bcatl(RNAi) worms. Color values in (b) represent relative intensities of features that have been $\log 2$-transformed and centered. c, Partial least squares - discriminant analysis. d, Pathway analysis using mummichog algorithm. e, TCA cycle metabolites/ precursors were decreased in bcat-1(RNAi) worms. $\mathrm{n}=6$ independent collections for control, 5 for bcat-1. f, Mitochondrial respiration was increased on day 5 in bcat-1 (RNAi) worms. $\mathrm{n}=10$ wells totaling 102 worms for control basal, 9 wells totaling 92 worms for control max., 10 wells totaling 100 worms per bcat-1 condition. g, Mitochondrial activity was increased on day 5 in CEP $\alpha$-synuclein-expressing dopaminergic neurons with bcat-1 knockdown. Scale bar, $10 \mu \mathrm{m}$. $\mathrm{n}=15$ worms totaling 47 CEPs for control, 11 worms totaling 39 CEPs for bcat-1. h, Protein carbonylation was increased on day 8 in CEP $\alpha$-synucleinexpressing dopaminergic neurons with bcat-1 knockdown. Scale bar, $10 \mu \mathrm{m} . \mathrm{n}=10$ worms totaling 37 CEPs for control, 10 worms totaling $38 \mathrm{CEPs}$ for bcat-1. Two-tailed $t$-tests. $* P<0.05$, $* * P<0.01, \quad * * * P<0.001, \quad * * * * P<0.0001$. Box-plots show minimum, 25th percentile, median, 75 th percentile, maximum.

expressed genes in these pathways were upregulated in bcat1(RNAi) neurons (Fig. 1i), suggesting that bcat-1 knockdown may promote a coordinated transcriptional response to alterations in BCAA metabolism.

\section{Metabolomics shows TCA cycle depletion while mitochondrial activity is increased}

To better understand the effects of bcat-1 knockdown on carbon metabolism, we next performed untargeted highresolution metabolomics ${ }^{18}$. Worms were fed bcat- 1 or control RNAi as adults and collected on day 5, the same timepoint as our transcriptomics analysis. Significantly altered features $(P<0.05)$ were detected in bcat-1 $(R N A i)$ worms compared with control worms (Fig. 2a,b, Extended Data Table 2), and PLS-DA showed distinct separation of the groups (Fig. 2c).

a

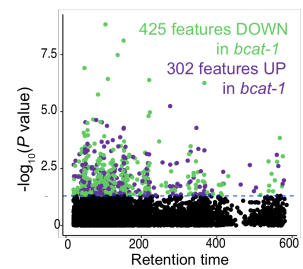

C

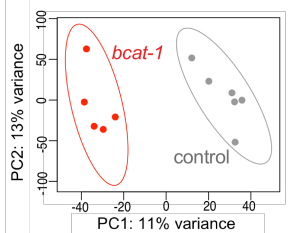

d

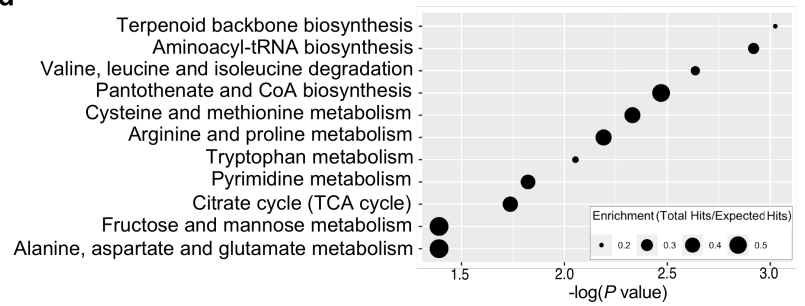

e

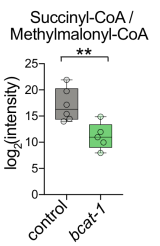

g

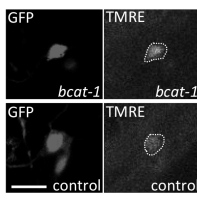

b

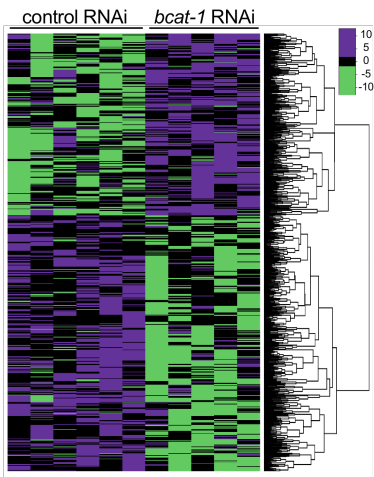

$\mathbf{f}$
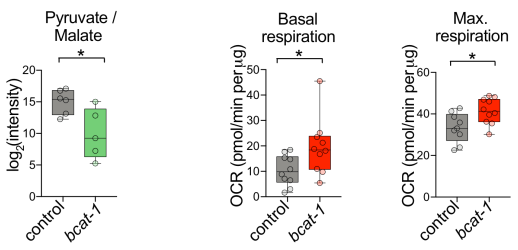

h

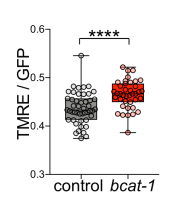

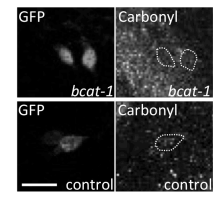

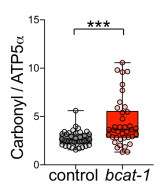


Mor et al., 2020 - preprint version -www.biorxiv.org

Several of the metabolic pathways that were significantly altered in bcat-1(RNAi) worms were related to BCAA metabolism, including valine, leucine, and isoleucine degradation, and alanine, aspartate, and glutamate metabolism (Fig. 2d). As expected, features putatively annotated as leucine/isoleucine and glutamate were increased or decreased, respectively, in bcat-1(RNAi) worms (Extended Data Fig. 4). Consistent with our transcriptomics analysis, citrate cycle (TCA cycle) was also a significantly altered metabolic pathway in bcat-1(RNAi) worms (Fig. 2d). However, while the expression of TCA cycle genes was largely increased (Fig. 1i), the levels of the annotated TCA cycle substrates and precursors pyruvate/malate and succinyl$\mathrm{CoA} /$ methylmalonyl-CoA were significantly decreased (Fig. 2e). These findings suggest that bcat-1 knockdown reduces steady-state levels of TCA cycle metabolites, while increasing expression of glycolysis and TCA cycle enzymes.

It is possible that low levels of TCA cycle metabolites leads to a reduction in mitochondrial respiration. Alternatively, low steady-state levels of metabolites may reflect more rapid TCA cycle turnover driven by more abundant components, resulting in higher levels of mitochondrial respiration. To distinguish between these possibilities, we measured mitochondrial activity in worms with bcat-1 knockdown. Oxygen consumption rates were assayed on day 5, and both basal and maximal respiration rates were found to be increased in bcat1(RNAi) worms (Fig. 2f). Similarly, using TMRE dye that accumulates only in active mitochondria, we found that dopaminergic neurons expressing $\alpha$-synuclein had increased mitochondrial activity with bcat-1 knockdown (Fig. 2g, Extended Data Fig. 5a). These data support the conclusion that bcat-1 reduction leads to mitochondrial hyperactivity.

Since mitochondrial respiration produces reactive oxygen species, we next tested for evidence of oxidative damage. Protein carbonylation levels were measured in $\alpha$-synucleinexpressing dopaminergic neurons with or without bcat-1 knockdown at the day 8 endpoint. Indeed, high mitochondrial activity was correlated with elevated levels of carbonylated proteins (Fig. 2h, Extended Data Fig. 5b), suggesting that disruption of bcat-1 dysregulates the cellular redox state such that neurons incur protein damage.

\section{Metformin reduces neurodegeneration and restores normal mitochondrial activity}

While disease mechanisms in PD are thought to involve decreased mitochondrial function ${ }^{19}$, our findings instead suggest that elevated mitochondrial activity may be a causative factor in PD. Strategies to restore mitochondrial homeostasis may therefore be efficacious. We recently identified the type 2 diabetes medication metformin as the topperforming candidate in a high-throughput screen for drugs that improve motor function in bcat-1(RNAi) worms ${ }^{3}$. Among

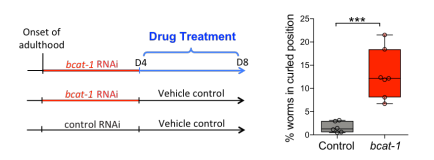

b
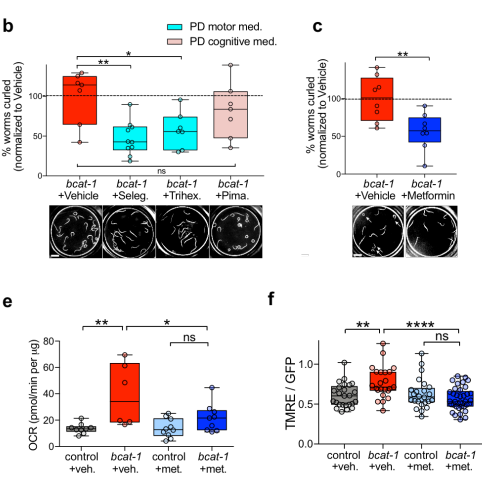

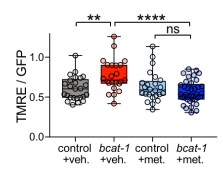

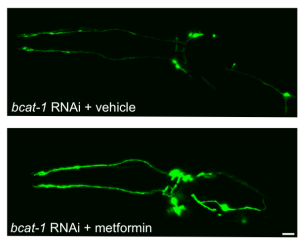

11 il
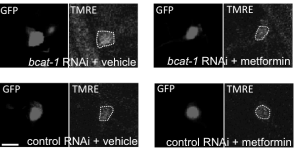

Figure 3: Metformin reduces neurodegeneration and restores normal mitochondrial activity in bcat-1(RNAi) worms. a, Experimental design for drug treatments. Neuronal RNAi-sensitive worms were fed bcat-1 or control RNAi as adults until day 4, then transferred to heat-killed OP50 E. coli and 50 $\mu \mathrm{M}$ drug or vehicle. Vehicle-treated bcat-1(RNAi) worms curled on day 8 , measured using our automated system. $\mathrm{n}=6$ wells totaling 91 worms for control, 7 wells totaling 57 worms for bcat-1. Two-tailed $t$-test. $\mathbf{b}$, PD medications prescribed for motor symptoms (selegiline (seleg.), trihexyphenidyl (trihex.)) reduced curling in bcat-1(RNAi) worms on day 8, whereas the antipsychotic pimavanserin (pima.) did not. Scale bar, $1 \mathrm{~mm} . \mathrm{n}=7$ wells totaling 142 worms for bcat-1, 10 wells totaling 149 worms for selegiline, 7 wells totaling 86 worms for trihexyphenidyl, 7 wells totaling 103 worms for pimavanserin. One-way ANOVA with Dunnett's post-hoc. c, Metformin reduced curling (arrows) on day 8 in bcat-1(RNAi) worms expressing $\alpha$-synuclein in dopaminergic neurons. Scale bar, $1 \mathrm{~mm}$. $\mathrm{n}=8$ wells totaling 65 worms for vehicle, 8 wells totaling 121 worms for metformin. Two-tailed $t$-test. d, Metformin reduced neurodegeneration of $\alpha$ synuclein-expressing dopaminergic neurons with bcat-1 knockdown on day 8 . Scale bar, $10 \mu \mathrm{m}$. $\mathrm{n}=11$ for CEP vehicle, 12 each for CEP and ADE metformin, 9 each for ADE and neurites vehicle, 10 for neurites metformin. Two-tailed $t$-tests. Data are mean \pm s.e.m. e, Metformin reduced basal mitochondrial respiration in bcat -1 (RNAi) worms on day $8 . \mathrm{n}=9$ wells totaling 144 worms for control vehicle, 6 wells totaling 50 worms for bcat-1 vehicle, 10 wells totaling 128 worms for control metformin, 9 wells totaling 113 worms for bcat- 1 metformin. Two-way ANOVA with Tukey's post-hoc. f, Metformin reduced mitochondrial activity in $\alpha$-synuclein-expressing CEP dopaminergic neurons with bcat-1 knockdown on day 6. Scale bar, $5 \mu \mathrm{m} . \mathrm{n}=8$ worms totaling 25 CEPs for control vehicle, 7 worms totaling 23 CEPs for bcat- 1 vehicle, 9 worms totaling 24 CEPs for control metformin, 10 worms totaling 37 CEPs for bcat-1 metformin. Two-way ANOVA with Tukey's post-hoc. met., metformin. veh., vehicle. ns, not significant. $* P<0.05$, $* * P<0.01, \quad * * * P<0.001, \quad * * * * P<0.0001$. Box-plots show minimum, 25th percentile, median, 75 th percentile, maximum. 
Mor et al., 2020- preprint version -www.biorxiv.org

several known targets, metformin appears to act as a complex I inhibitor ${ }^{20}$, raising the possibility that reducing mitochondrial activity may constitute a surprising new treatment avenue for PD.

To investigate the potential neuroprotective action of metformin and its effects on mitochondria in the bcat-l PD worm model, we used the experimental paradigm established in our high-throughput drug screen ${ }^{3}$. Neuronal RNAi-sensitive worms were fed bcat- 1 or control RNAi as adults, and on day 4 transferred to plates seeded with heat-killed OP50 E. coli and $50 \mu \mathrm{M}$ drug or vehicle $(0.05 \%$ DMSO) (Fig. 3a). The use of heat-killed bacteria eliminates potential confounding effects of bacterial metabolism of the drugs, as has been documented for metformin ${ }^{21}$. In addition, drug intervention on day 4 mimics the current clinical landscape, in which PD diagnosis and treatment occurs well into the disease course ${ }^{22}$. On day 8 , curling was quantified using our automated system ${ }^{3}$. Exposure to bcat-1 RNAi until day 4, followed by vehicle treatment was sufficient to produce significant curling on day 8 (Fig. 3a). Using this paradigm, two PD medications currently prescribed for motor symptoms significantly reduced curling, whereas a PD drug indicated for cognitive symptoms had no effect (Fig. $3 b$ ), supporting the notion that curling reflects the motility defects of PD.

To determine if metformin rescues bcat-1-associated toxicity in worms with $\alpha$-synuclein expression in dopaminergic neurons, we measured motor function and dopamine neuron degeneration. Worms treated with $50 \mu \mathrm{M}$ metformin as described (Fig. 3a) showed reduced curling on day 8 (Fig. 3c). Remarkably, these worms also had significantly less neurodegeneration. On day 8 , the number of dopaminergic cell bodies and neurites was increased in metformin-treated bcat1(RNAi) worms compared with vehicle-treated worms (Fig. $3 \mathrm{~d})$. Neuroprotection was detected as early as day 6 , after only two days of metformin treatment (from day 4 to day 6), in that dopaminergic neurons showed significantly improved neurite morphologies (Extended Data Fig. 6).

We next asked if metformin might exert its protective effects by suppressing bcat-1(RNAi)-driven mitochondrial hyperactivity. Indeed, we found on day 8 that metformin treatment had reduced both basal and maximal mitochondrial respiration down to levels of age-matched controls (Fig. 3e, Extended Data Fig. 7a). Similarly, using TMRE dye on day 6 (the latest timepoint permitted by the assay), metformin treatment was already sufficient to restore mitochondrial activity levels in $\alpha$-synuclein-expressing dopaminergic neurons of bcat-1(RNAi) worms (Fig. 3f, Extended Data Fig. $7 b)$. These data suggest that metformin is able to correct aberrant mitochondrial activity in the bcat-1(RNAi) worm model of PD.

\section{Conclusions}

Here we demonstrate that defective BCAA metabolism recapitulates several major features of $\mathrm{PD}$, including motor dysfunction and neurodegeneration. Despite an intriguing overlap of clinical and pathological features between BCAA metabolic disorders and $\mathrm{PD}^{23}$, a potential link remains largely unexplored. Maple syrup urine disease (MSUD) arises from mutations in BCKDHC subunits, and commonly presents with parkinsonian symptoms in adult patients ${ }^{24}$. Strikingly, neuronal loss in the substantia nigra has also been documented in MSUD ${ }^{25}$. Meta-analysis of genome-wide association studies revealed associations between $\mathrm{PD}$ and the BCAA pathway genes methylcrotonoyl-CoA carboxylase 1 (MCCC1) and branched-chain ketoacid dehydrogenase kinase $(B C K D K)^{26}$, further implicating this pathway in disease risk. Consistent with this, metabolomic profiling of PD patient urine revealed that BCAAs were elevated relative to controls, and higher BCAA levels were correlated with greater disease severity ${ }^{27}$.

Our transcriptomics, metabolomics, and respiration experiment approaches led to the surprising finding that $b c a t-1$ reduction causes mitochondrial hyperactivity, consistent with reports from mice lacking the mitochondrial $B C A T$ isoform ${ }^{28}$. These findings challenge the prevailing notion that mitochondrial function is solely decreased in $\mathrm{PD}$, in particular due to complex I deficiency, which has been documented in peripheral tissues from PD patients ${ }^{29}$ and postmortem patient brain samples ${ }^{30}$. Critically, however, mitochondrial activity levels in PD patient brain prior to death remain unknown, and therefore it is possible that an increase in activity precedes the ultimate loss of function. Complex I inhibitors such as MPTP and rotenone are often used to model PD, and MPTP induces parkinsonism in humans ${ }^{31}$. However, MPTP produces acute neurodegeneration that does not model the progressive nature of $\mathrm{PD}^{32}$, and rotenone models suffer from issues of variability and subject mortality ${ }^{33}$. While these compounds may model endpoint processes in the disease, our study offers mitochondrial hyperactivity as a potential early pathological event in PD.

In support of the notion that hyperactive mitochondria may drive disease and therefore reducing activity levels back to normal may be protective, we found that metformin treatment restored normal mitochondrial activity and reduced both neurodegeneration and motor deficits in a model of PD driven by defective BCAA metabolism. Collectively, our work supports a metabolic origin of $\mathrm{PD}$, and points to metformin as an existing FDA-approved drug with great promise for repurposing to $\mathrm{PD}$. 
Mor et al., 2020 - preprint version -www.biorxiv.org

\section{Methods}

C. elegans strains and maintenance. Worms were maintained at $20^{\circ} \mathrm{C}$ on standard nematode growth medium (NGM) plates or high growth medium (HG) plates, seeded with OP50 Escherichia coli or HT115 RNAi Escherichia coli, as indicated. The following strains were used in this study: wild-type worms of the N2 Bristol strain, neuron-only RNAi strain CQ511 [sid-1(pk3321)]; uIs69 [pCFJ90 (myo$2 p:: m$ Cherry, unc-119p::sid-1)], and the following neuronal RNAi-sensitive strains, CF512 (fem-1(hcl7); fer-15(b26)),

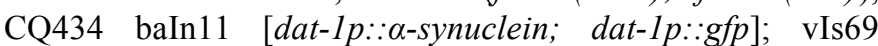
[pCFJ90 (myo-2p::mCherry + unc-119p::sid-1)], and TU3311 uIs60 (unc-119p::sid-1, unc-119p::yfp).

RNAi and drug treatments. Worms were synchronized from eggs by bleaching and plated onto HG plates seeded with OP50. At the L4 stage, worms were transferred to RNAiseeded $100-\mathrm{mm}$ NGM or HG plates containing carbenicillin and IPTG that were pre-induced with $0.1 \mathrm{M}$ IPTG 1 hour prior to transfer. Worms were transferred to fresh RNAi plates every 2-3 days. In all experiments, control RNAi refers to empty vector pL4440 in HT115 Escherichia coli. For experiments with metformin or PD drugs, worms were transferred on day 4 to NGM plates seeded with $1 \mathrm{~mL}$ heatkilled OP50 bacteria and $50 \mu \mathrm{M}$ metformin (Sigma), $50 \mu \mathrm{M}$ selegiline (MedChem Express), $50 \mu \mathrm{M}$ trihexyphenidyl (MedChem Express), $50 \mu \mathrm{M}$ pimavanserin (MedChem Express), or vehicle (0.5\% DMSO). OP50 was killed by incubation at $65^{\circ} \mathrm{C}$ for $30 \mathrm{~min}$. The worms were transferred to fresh NGM plates with $1 \mathrm{~mL}$ heat-killed OP50 and drug or vehicle on day 6. For rapamycin experiments, rapamycin (LC Laboratories) was prepared in NGM media at a final concentration of $100 \mu \mathrm{M}$, and worms were maintained on rapamycin or vehicle plates from L4 to day 8 with transfer to fresh plates every 2-3 days.

Curling quantification. Manual quantification of individual worms' curling level was performed as previously described ${ }^{2}$, and defined as percentage of time spent curling. We recently developed an automated platform for high-throughput curling analysis ${ }^{3}$. Briefly, on the day of analysis, worms were washed twice rapidly with M9 buffer and dispensed into 96-well plates. 30-sec videos or a series of snapshots were obtained for individual wells containing 3-30 worms each. Our curling detection software quantified the \# of worms in a curled position, divided by the total \# of worms detected, and this was defined as percentage of worms in a curled position.

Neurodegeneration assays. Animals were mounted on $2 \%$ agarose pads in M9 and sodium azide. For dopaminergic (datlp::GFP-labeled) neurons, scoring was done as previously described $^{2}$. Briefly, images were obtained on a Nikon A1 confocal microscope at 40x magnification, and $\mathrm{z}$ stacks were processed in Nikon NIS elements software. CEP and ADE cell bodies were counted, as well as neurites projecting anteriorly from CEP cell bodies.

Basal slowing behavioral assays. On day 5, CQ434 baIn11

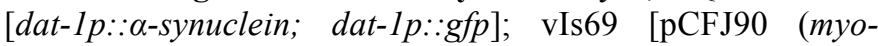
$2 p:: m$ Cherry + unc-119p::sid-1)] worms were individually picked onto NGM plates seeded with OP50. After $2 \mathrm{~min}$, the number of body bends was manually counted for $20 \mathrm{sec}$. Each worm was then picked onto an unseeded NGM plate. After 2 $\mathrm{min}$, the number of body bends was manually counted for 20 $\mathrm{sec}$. If a worm curled during a $20 \mathrm{sec}$ measurement period, the $20 \mathrm{sec}$ was restarted when the worm resumed normal movement. The assay could not be performed on day 6 due to excessive curling.

Neuron isolation and transcriptomics. Adult neuron isolation was performed as described previously ${ }^{14}$. Briefly, TU3311 uIs60 (unc-119p::sid-1, unc-119p::yfp) worms were synchronized by bleaching and transferred to fresh bcat-1 or control RNAi plates every 2-3 days starting at L4. Adult worms were separated from progeny every 2 days by sedimentation in M9 buffer. On day 5, worms were washed with M9 buffer and incubated for $6.5 \mathrm{~min}$ with lysis buffer (200 mM DTT, 0.25\% SDS, $20 \mathrm{mM}$ Hepes pH 8.0, 3\% sucrose). The worms were washed with M9 buffer and incubated in $20 \mathrm{mg} / \mathrm{ml}$ pronase from Streptomyces griseus (Sigma) for 12-20 min with vigorous pipetting every 2-3 min. When worm bodies were dissociated and the solution became cloudy, $2 \%$ fetal bovine serum (Gibco) was added to stop the reaction, and the mixture was filtered through $5 \mu \mathrm{m}$ filters (Millipore). GFP-labeled neurons were sorted using a FACSVantage SE w/ DiVa (BD Biosciences; 488nm excitation for GFP detection), with age-matched N2 worms used a negative control for setting gates. RNA was extracted, DNase digested, and cleaned using Qiagen RNEasy Minelute columns as previously described ${ }^{14,15}$. RNA sequencing libraries were prepared using the SMARTer Stranded Total RNA kit v2-Pico input mammalian, as per manufacturer instructions. Libraries were pooled and sequenced on the Illumina HiSeq 2000 platform, and the Galaxy Workflow System was used to analyze the RNA sequencing data. Reads were mapped to the C. elegans genome (WS245) using RNA STAR, and mapped reads that overlap with gene features were counted using htseq-count (mode $=$ union). Per-gene counts were used as input for the DESeq2 $\mathrm{R}$ package for differential gene expression analysis. Five total independent collections were obtained for each condition (bcat-1 or control), and bcat1 curling was confirmed for each replicate on day 8. Upon publishing, raw sequencing reads will be made available at NCBI Bioproject: PRJNA599166.

Gene Ontology and KEGG analyses. Lists of up- or downregulated genes in bcat-1(RNAi) neurons were analyzed using gProfiler ${ }^{34}$ with the following settings: $C$. elegans organism, only annotated genes, g:SCS threshold, user 
threshold .05, ENTREZGENE_ACC. REVIGO was used to cluster and plot GO terms with q-value $<0.05$.

High-resolution metabolomics. CF512 (fem-1(hc17); fer15(b26)) worms were synchonized by bleaching and sterilized by incubation at $25^{\circ} \mathrm{C}$ from L2-L4. Worms were transferred to fresh bcat-1 or control RNAi plates every 2-3 days starting at L4. Adult worms were separated from progeny every 2 days by sedimentation in M9 buffer. On day 5, worms were washed with M9 buffer and $\sim 500$ worms were snap frozen in liquid nitrogen per replicate. In parallel for each sample, $\sim 500$ worms were incubated in M9 for 1 hour to release gut bacteria, and then the starved worms were discarded and the bacteria were collected as a blank. Metabolites were extracted using acetonitrile (in a 2:1 ratio) which was added to all samples along with an internal standard. Bead-beating was used to disrupt the worm cuticle and improve extraction. Each sample was placed in the bead beater at speed $6.5 \mathrm{~m} / \mathrm{s}$ for 30 seconds, allowed to equilibrate on ice for one minute, and placed in the beater for another 30 seconds, at the same speed. All processing was done either on ice or in a cold room. Nontargeted high-resolution mass spectrometry was run at the Clinical Biomarkers lab at Emory University using a HILIC column (positive mode) and C18 column (negative mode) using chromatographic methods previously described ${ }^{35}$. Mass spectral data was generated on an orbitrap mass spectrometer on full scan mode scanning for mass range 85 to $850 \mathrm{Da}$. Data were extracted using the $\mathrm{R}$ packages apLCMS ${ }^{36}$ and xMSanalyzer $^{37}$. Features that were 1.5 times the intensity in the respective bacterial blank and were present in at least 9 of the 21 samples were further analyzed. Features were imputed if missing with half the value of the minimum abundance, normalized to protein content of the sample, and $\log 2$ transformed. All data processing, analysis and visualization was done in $\mathrm{R}$ version 3.6.0, using functions: gplots::heatmap2( $)^{38}, \quad$ ggplot2:: $\operatorname{ggplot}()^{39}, \quad$ and mixOmics::plsda ()$^{40}$. Pathway analysis was done using the mummichog algorithm ${ }^{41}$ hosted on the metaboanlayst (www.metaboanalyst.ca) module "MS peaks to Pathway",42, using the Caenorhabditis elegans metabolic reference map available through KEGG. Five total independent collections were obtained for bcat-1(RNAi) and six independent collections were obtained for control RNAi. bcat-1 curling was confirmed for each replicate on day 8 .

Oxygen consumption measurements. Whole-worm respiration was measured using a Seahorse XFe96 analyzer as previously described ${ }^{43}$. Briefly, worms were plated (5-25 worms per well) in $200 \mu \mathrm{L}$ M9 buffer containing dilute heatkilled OP50 to prevent starvation. The same dilute heat-killed OP50 solution was used as a blank. Baseline respiration was measured, followed by injection of FCCP $(10 \mu \mathrm{M}$, final concentration) to elicit maximal respiration, followed by sodium azide ( $40 \mathrm{mM}$, final concentration) to account for non- mitochondrial respiration. Measurements were normalized to protein content per well, determined by BCA assay.

TMRE staining. NGM plates seeded with $1 \mathrm{~mL}$ heat-killed OP50 were spotted with tetramethylrhodamine ethyl ester (TMRE) to a final concentration of $30 \mu \mathrm{M}$ and allowed to dry. Worms were transferred to TMRE plates, and the following day, animals were mounted on $2 \%$ agarose pads with levamisole. Images of dopaminergic cell bodies were obtained on a Nikon A1 confocal microscope at 100x magnification, and $\mathrm{z}$ stacks were processed in Nikon NIS elements software. For each cell, a region of interest was drawn around the cell body at the plane with the greatest GFP intensity. The corresponding TMRE intensity (TRITC channel) was normalized to the GFP intensity.

Protein carbonylation staining. Staining was performed using the OxyBlot kit as previously described ${ }^{44}$. Briefly, worms were washed $3 \mathrm{x}$ with M9 and incubated in $1 \mathrm{X}$ DNPH for $30 \mathrm{~min}$ at room temperature. The reaction was blocked by adding $4 \mathrm{x}$ volume of neutralization buffer. Worms were washed $2 \mathrm{x}$ with ice-cold water, sandwiched between two polyL-lysine coated slides, and incubated for $20 \mathrm{~min}$ in liquid nitrogen. The slide sandwiches were then freeze-cracked, the worms were fixed with methanol followed by acetone, and then blocked for one hour at room temperature with 1X PBS, $0.2 \%$ Gelatine, $0.25 \%$ Triton X-100. The primary antibody was applied at 1:100 dilution in blocking solution and incubated overnight at $4^{\circ} \mathrm{C}$. As an internal control, antiATP5A antibody was co-incubated. Following four 10-min washed with $0.25 \%$ Triton X-100 in 1X PBS, secondary antibody was applied at 1:300 dilution in blocking solution and incubated overnight at $4^{\circ} \mathrm{C}$. Images of dopaminergic cell bodies were obtained on a Nikon A1 confocal microscope at 60x magnification, and $\mathrm{z}$ stacks were processed in Nikon NIS elements software. For each cell, a region of interest was drawn around the cell body at the plane with the greatest GFP intensity. The corresponding anti-DNPH intensity (TRITC channel) was normalized to the anti-ATP5A intensity (Cy5 channel).

Statistical analysis. For all comparisons between two groups, an unpaired two-tailed Student's $t$-test was performed. For comparisons between multiple groups, One-Way ANOVA or Two-Way ANOVA (for 2 variables, i.e. RNAi treatment and +/- metformin) were performed with post-hoc testing as indicated. GraphPad Prism was used for all statistical analyses.

Acknowledgments: We thank the C. elegans Genetics Center for strains (P40 OD010440), the Genomics Core Facility and Confocal Imaging Facility at Princeton University, Dr. Dean Jones and the Clinical Biomarkers Laboratory at Emory University, A. Tartici for help with data analysis, and the 
Mor et al., 2020 - preprint version -www.biorxiv.org

Murphy lab for discussion. C.T.M. is the Director of the Glenn Center for Aging Research at Princeton and an HHMI-Simons Faculty Scholar. D.E.M. was supported by Ruth L. Kirschstein NRSA (NIA F32AG062036) and further support was provided by NIH (NIEHS U2CES030163 and R01ES023839) to G.W.M., and NIH DP1 Pioneer Award to C.T.M. (NIGMS 5DP1GM119167) and The Glenn Foundation for Medical Research to C.T.M. (GMFR CNV1001899).

Author Contributions: Conceptualization, D.E.M., R.K., and C.T.M.; Methodology, D.E.M., S.S., R.K., W.K., V.K., G.W.M., and C.T.M.; Investigation, D.E.M., S.S., R.K., W.K., and V.K.; Writing - Original Draft, D.E.M.; Writing - Review \& Editing, D.E.M., R.K., V.K., G.W.M., and C.T.M.; Funding Acquisition, D.E.M., G.W.M., and C.T.M.

Declaration of Interests: The authors declare no competing interests.

\section{References}

1. Cai H., et al. (2012) Metabolic dysfunction in Alzheimer's disease and related neurodegenerative disorders. Curr Alzheimer Res 9(1):5-17.

2. Yao V. \& Kaletsky R., et al. (2018) An integrative tissuenetwork approach to identify and test human disease genes. Nature Biotechnology 36:1091-99.

3. Sohrabi S., et al. High-throughput behavioral screen in $C$. elegans reveals novel Parkinson's drug candidates, in preparation.

4. Van Den Eeden S.K., et al. (2003) Incidence of Parkinson's disease: variation by age, gender, and race/ethnicity. Am J Epidemiol 157:1015-22.

5. de Lau L.M. \& Breteler M.M. (2006) Epidemiology of Parkinson's disease. Lancet Neurol 5:525-35.

6. Forno L.S. (1996) Neuropathology of Parkinson's disease. J Neuropathol Exp Neurol 55:259-72.

7. Spillantini M.G., et al. (1997) Alpha-synuclein in Lewy bodies. Nature 388:839-40.

8. Klein C. \& Westenberger A. (2012) Genetics of Parkinson's Disease. Cold Spring Harb Perspect Med 2, a008888-a008888.

9. Valerio A., et al. (2011) Branched-chain amino acids, mitochondrial biogenesis, and healthspan: an evolutionary perspective. Aging (Albany NY) 3(5):464-78.

10. Corsi A.K., et al. (2015) A Transparent window into biology: A primer on Caenorhabditis elegans. WormBook 1-31. http://www.wormbook.org.

11. Rual J.F., et al., (2004) Toward improving Caenorhabditis elegans phenome mapping with an ORFeome-based RNAi library. Genome Res 14(10B):2162-8.

12. Sawin E.R., et al. (2000) C. elegans locomotory rate is modulated by the environment through a dopaminergic pathway and by experience through a serotonergic pathway. Neuron 26:619-631.
13. Mansfeld J., et al. (2015) Branched-chain amino acid catabolism is a conserved regulator of physiological ageing. Nat Commun 6:10043.

14. Kaletsky R., et al. (2016) The C. elegans adult neuronal IIS/FOXO transcriptome reveals adult phenotype regulators. Nature 529:92-6.

15. Kaletsky R., et al. (2018) Transcriptome Analysis of Adult Caenorhabditis elegans Cells Reveals Tissuespecific Gene and Isoform Expression. PLoS Genet 14(8):e1007559.

16. Watson E., et al. (2016) Metabolic network rewiring of propionate flux compensates vitamin B12 deficiency in C. elegans. Elife 5 pii: e17670.

17. Meissner B., et al. (2011) Determining the sub-cellular localization of proteins within Caenorhabditis elegans body wall muscle. PLoS One 6(5):e19937.

18. Go Y.M., et al. (2015) Reference Standardization for Mass Spectrometry and High-resolution Metabolomics Applications to Exposome Research. Toxicol Sci 148(2):531-43.

19. Winklhofer K.F. \& Haass C. (2010) Mitochondrial dysfunction in Parkinson's disease. Biochim Biophys Acta. 1802(1):29-44.

20. Vial G., et al. (2019) Role of Mitochondria in the Mechanism(s) of Action of Metformin. Front Endocrinol (Lausanne) 10:294.

21. Cabreiro F., et al. (2013) Metformin retards aging in C. elegans by altering microbial folate and methionine metabolism. Cell 153(1):228-39.

22. Cheng H.C., et al. (2010) Clinical progression in Parkinson disease and the neurobiology of axons. Ann Neurol 67:715-725.

23. Limphaibool N., et al. (2018) Parkinsonism in Inherited Metabolic Disorders: Key Considerations and Major Features. Front Neurol 9:857.

24. Carecchio M., et al. (2011) Movement disorders in adult surviving patients with maple syrup urine disease: Movement Disorders in Adult MSUD Patients. Mov Disord 26, 1324-1328.

25. Kiil R. \& Rokkones T. (1964) Late manifesting variant of branched-chain ketoaciduria (maple syrup urine disease). Acta Paediatr 53:356-64.

26. Nalls M.A., et al. (2014) Large-scale meta-analysis of genome-wide association data identifies six new risk loci for Parkinson's disease. Nat Genet 46(9):989-93.

27. Luan H., et al. (2015) Comprehensive urinary metabolomic profiling and identification of potential noninvasive marker for idiopathic Parkinson's disease. Sci Rep 5, 13888.

28. She P., et al. (2007) Disruption of BCATm in mice leads to increased energy expenditure associated with the activation of a futile protein turnover cycle. Cell Metab 6(3):181-94.

29. Parker, W.D., et al. (1989) Abnormalities of the electron transport chain in idiopathic Parkinson's disease. Ann Neurol 26, 719-723. 
bioRxiv preprint doi: https://doi.org/10.1101/2020.02.20.958322; this version posted February 22, 2020. The copyright holder for this preprint (which was not certified by peer review) is the author/funder. All rights reserved. No reuse allowed without permission.

Mor et al., 2020- preprint version -www.biorxiv.org

30. Schapira A.H., et al. (1989) Mitochondrial complex I deficiency in Parkinson's disease. Lancet 1(8649):1269.

31. Dauer W. \& Przedborski S. (2003) Parkinson's disease: mechanisms and models. Neuron 39:889-909.

32. Tieu K. (2011) A guide to neurotoxic animal models of Parkinson's disease. Cold Spring Harb Perspect Med 1:a009316.

33. Cicchetti F., et al. (2009) Environmental toxins and Parkinson's disease: what have we learned from pesticide-induced animal models? Trends Pharmacol Sci 30(9):475-83.

34. Raudvere U., et al. (2019) g:Profiler: a web server for functional enrichment analysis and conversions of gene lists (2019 update) Nucleic Acids Research doi:10.1093/nar/gkz369.

35. Liu K.H., et al. (2016) High-resolution Metabolomics Assessment of Military Personnel: Evaluating Analytical Strategies for Chemical Detection. Journal of Occupational and Environmental Medicine 58.

36. Yu T., et al. (2009) apLCMS-adaptive processing of high-resolution LC/MS data. Bioinformatics 25, 19301936.

37. Uppal K., et al. (2013) xMSanalyzer: automated pipeline for improved feature detection and downstream analysis of large-scale, non-targeted metabolomics data. BMC Bioinformatics 14, 15.

38. Warnes G.R., et al. (2019) gplots: Various R Programming Tools for Plotting Data. R package version 3.0.1.1. https://CRAN.R-project.org/package=gplots

39. Wickham H. ggplot2: Elegant Graphics for Data Analysis. Springer-Verlag New York, 2016.

40. Le Cao K., et al. (2016) mixOmics: Omics Data Integration Project. $\mathrm{R}$ package version 6.1.1. https://CRAN.R-project.org/package=mixOmics

41. Li S., et al. (2013) Predicting Network Activity from High Throughput Metabolomics. PLOS Computational Biology 9, e1003123.

42. Chong J., et al. (2019) Using MetaboAnalyst 4.0 for Comprehensive and Integrative Metabolomics Data Analysis. Current Protocols in Bioinformatics 68, e86.

43. Koopman M., et al. (2016) A screening-based platform for the assessment of cellular respiration in Caenorhabditis elegans. Nat Protoc 11(10):1798-816.

44. Goudeau J. \& Aguilaniu H. (2010) Carbonylated proteins are eliminated during reproduction in C. elegans. Aging Cell 9(6):991-1003.

\section{Extended Data Figures and Tables}

a
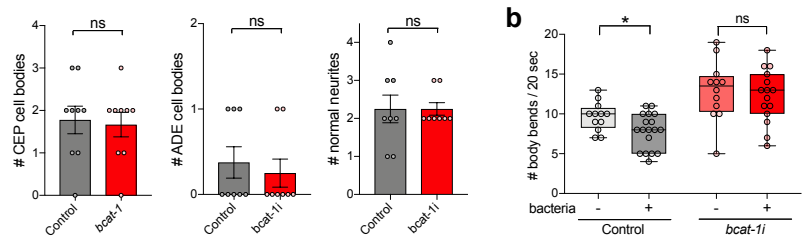

Extended Data Fig. 1: a, $\alpha$-synuclein-expressing dopaminergic neurons with or without bcat-1 knockdown are largely degenerated by day $8 . \mathrm{n}=9$ each for control and bcat -1 CEP, 8 each for control and bcat-1 ADE and neurites. Two-tailed $t$-tests. Data are mean \pm s.e.m. b, While dopaminergic-dependent basal slowing behavior is still intact on day 5 in worms expressing $\alpha$-synuclein in dopaminergic neurons, it is absent with additional knockdown of bcat-1. $\mathrm{n}=12$ each for (-)bacteria, 18 for control (+)bacteria, 15 for bcat- $1(+)$ bacteria. Two-tailed $t$-tests. ns, not significant. $* P<0.05$. Box-plots show minimum, 25th percentile, median, 75 th percentile, maximum.

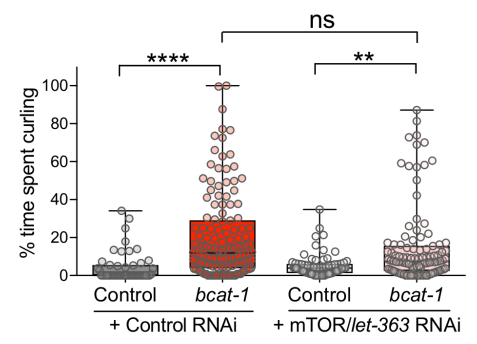

Extended Data Fig. 2: Curling is unaffected by mTOR/let363 knockdown in neuronal RNAi-sensitive worms with bcat-1 knockdown on day $8 . \mathrm{n}=48$ for control, 134 for bcat -1 control, 72 for control let-363, 106 for bcat-1 let-363. Two-way ANOVA with Tukey's post-hoc. ns, not significant. ${ }^{* *} P<0.01, * * * * P<$ 0.0001 . Box-plots show minimum, 25 th percentile, median, 75 th percentile, maximum. 


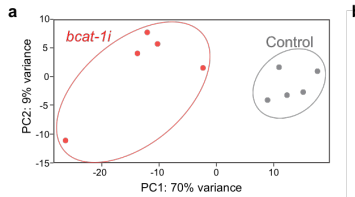

d
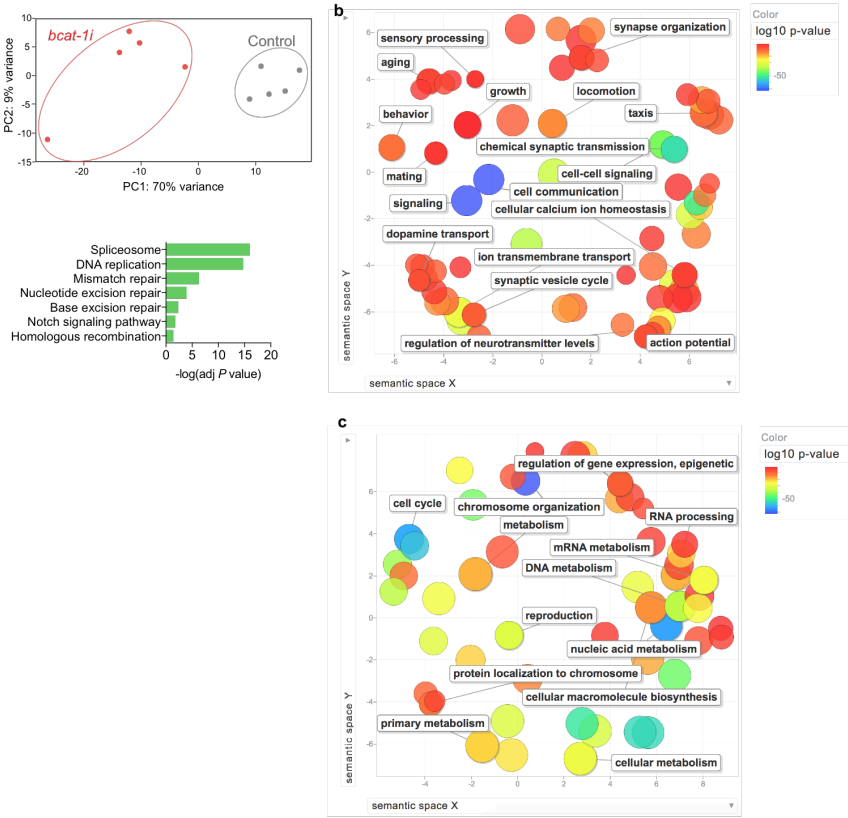

Extended Data Fig. 3: a, PCA plot of neuronal transcriptomes from worms with bcat-1 knockdown and controls on day 5 . b-c, Gene Ontology analysis of significantly upregulated (b) and downregulated (c) genes in bcat-1(RNAi) neurons. d, KEGG pathway analysis of significantly downregulated genes in bcatl(RNAi) neurons. FDR $<0.05 . \mathrm{n}=5$ independent collections/group.

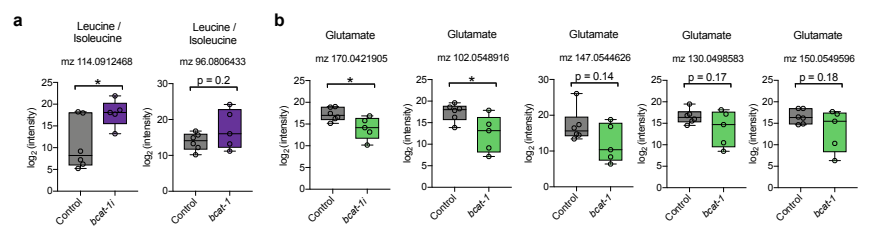

Extended Data Fig. 4: a-b, Levels of features annotated by mummichog as leucine/isoleucine (a) or glutamate (b) in bcat1(RNAi) worms and controls. $\mathrm{n}=6$ independent collections for control, 5 for $b c a t$ - 1 . Two-tailed $t$-tests. $* P<0.05$. Box-plots show minimum, 25th percentile, median, 75 th percentile, maximum.

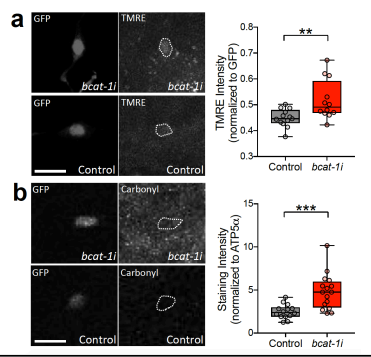

Extended Data Fig. 5: a, Mitochondrial activity was increased on day 5 in ADE $\alpha$-synuclein-expressing dopaminergic neurons with bcat-1 knockdown. Scale bar, $10 \mu \mathrm{m}$. $\mathrm{n}=13$ worms totaling 13 ADEs for control, 11 worms totaling 12 ADEs for bcat-1. b, Protein carbonylation was increased on day 8 in ADE $\alpha$-synuclein-expressing dopaminergic neurons with bcat-1 knockdown. Scale bar, $10 \mu \mathrm{m} . \mathrm{n}=10$ worms totaling 14 ADEs for control, 10 worms totaling 16 ADEs for bcat-1. Two-tailed $t$ tests. $* * P<0.01, * * * P<0.001$. Box-plots show minimum, 25th percentile, median, 75 th percentile, maximum.
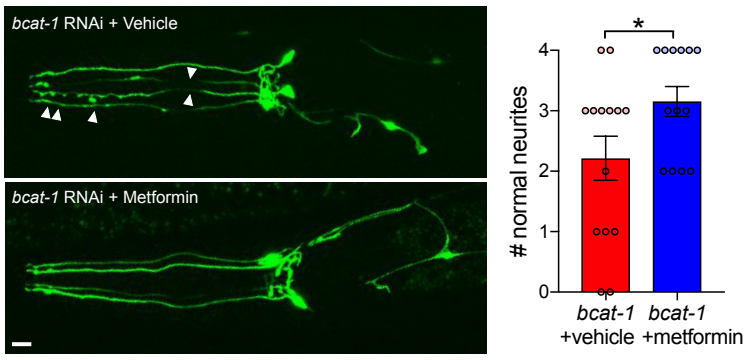

Extended Data Fig. 6: Metformin reduced degenerated morphologies (blebbing and fragmentation, arrowheads) in $\alpha$ synuclein-expressing dopaminergic neurites with bcat-1 knockdown on day 6 , following only two days of treatment. Scale bar, $10 \mu \mathrm{m} . \mathrm{n}=14$ for vehicle, 13 for metformin. Two-tailed $t$-test. Data are mean \pm s.e.m.
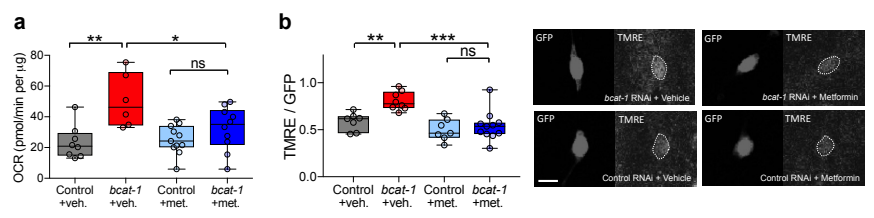

Extended Data Fig. 7: a, Metformin reduced maximal mitochondrial respiration in bcat-1 (RNAi) worms on day $8 . \mathrm{n}=8$ wells totaling 119 worms for control vehicle, 6 wells totaling 50 worms for bcat-1 vehicle, 11 wells totaling 139 worms for control metformin, 10 wells totaling 123 worms for bcat-1 metformin. Two-way ANOVA with Tukey's post-hoc. b, Metformin reduced mitochondrial activity in $\alpha$-synucleinexpressing ADE dopaminergic neurons with bcat-1 knockdown on day 6 . Scale bar, $5 \mu \mathrm{m}$. $\mathrm{n}=7$ worms totaling 7 ADEs for control vehicle, 7 worms totaling 8 ADEs for bcat- 1 vehicle, 7 worms totaling 7 ADEs for control metformin, 10 worms totaling 11 ADEs for bcat- 1 metformin. Two-way ANOVA with Tukey's post-hoc. ns, not significant. $* P<0.05, * * P<0.01, * * * P<0.001$. Box-plots show minimum, 25th percentile, median, 75th percentile. maximum. 
Extended Data Table 1: Differentially-expressed genes in bcat-1(RNAi) neurons. Lists of all genes significantly up- or downregulated in bcat-1(RNAi) neurons (FDR $<0.05)$, as well as subsets of each that were previously identified as neuronally-expressed ${ }^{15}$.

Extended Data Table 2: Annotated features significant at $\mathbf{p}<0.05$ from metabolomics analysis of bcat-1(RNAi) worms. m.z, mass-to-charge ratio; retention time, retention time off of the column; KEGG.ID, KEGG ID determined by mummichog hosted on metaboanalyst; Annotation, Compound name from KEGG. (Level 5 confidence in annotation (Schymanksi 2014)); Adduct, Metabolite adduct annotated; Mass.Diff, Difference in mass of adduct detected and true mass of adduct. 